OPEN ACCESS

Edited by:

Xuping Li,

Houston Methodist Research

Institute, United States

Reviewed by:

Maria Angeles Arevalo,

Spanish National Research Council (CS/C), Spain

Gyun Jee Song,

Catholic Kwandong University,

South Korea

*Correspondence:

Eugene D. Ponomarev

eponomarev@cuhk.edu.hk;

eugpon@gmail.com

Specialty section:

This article was submitted to

Non-neuronal Cells,

a section of the journal

Frontiers in Cellular Neuroscience

Received: 14 June 2019

Accepted: 23 September 2019

Published: 11 October 2019

Citation:

Veremeyko T, Yung AWY,

Dukhinova M, Strekalova T and

Ponomarev ED (2019) The Role of Neuronal Factors in the Epigenetic

Reprogramming of Microglia in the Normal and Diseased Central

Nervous System.

Front. Cell. Neurosci. 13:453.

doi: $10.3389 /$ fncel.2019.00453

\section{The Role of Neuronal Factors in the Epigenetic Reprogramming of Microglia in the Normal and Diseased Central Nervous System}

\author{
Tatyana Veremeyko ${ }^{1}$, Amanda W. Y. Yung ${ }^{1}$, Marina Dukhinova ${ }^{1,2}$, Tatyana Strekalova ${ }^{3,4,5}$ \\ and Eugene D. Ponomarev ${ }^{1,6 *}$
}

\begin{abstract}
${ }^{1}$ School of Biomedical Sciences, Faculty of Medicine, The Chinese University of Hong Kong, Shatin, Hong Kong, ${ }^{2}$ Synthetic and Systems Biology for Biomedicine, Italian Institute of Technology (IIT), Genoa, Italy, ${ }^{3}$ Department of Psychiatry and Neuropsychology, Maastricht University, Maastricht, Netherlands, ${ }^{4}$ Institute of General Pathology and Pathophysiology, Moscow, Russia, ${ }^{5}$ Laboratory of Psychiatric Neurobiology and Department of Normal Physiology, Institute of Molecular Medicine, Sechenov First Moscow State Medical University, Moscow, Russia, ${ }^{6}$ Kunming Institute of Zoology, Chinese University of Hong Kong Joint Laboratory of Bioresources and Molecular Research in Common Diseases, Chinese Academy of Sciences, Kunming, China
\end{abstract}

Twenty years ago, the scientific community exhibited relatively little interest in the study of microglial cells. However, recent technical and conceptual advances in this field have greatly increased interest in the basic biology of these cells within various neurodegenerative diseases, including multiple sclerosis, Alzheimer's disease, and traumatic brain/spinal cord injuries. The main functions of these cells in the normal central nervous system (CNS) remain poorly understood, despite considerable elucidation of their roles in pathological conditions. Microglia populate the brain before birth and remain in close lifelong contact with CNS-resident cells under the influence of the local microenvironment. Within the CNS parenchyma, microglia actively interact with two main cell types, astrocytes and neurons, which produce many factors that affect microglia phenotypes in the normal CNS and during neuroinflammation. These factors include interleukin (IL)-34, macrophage colony-stimulating factor, transforming growth factor- $\beta$, and IL-4, which promote microglial expansion, survival, and differentiation to an anti-inflammatory phenotype in the normal CNS. Under inflammatory conditions, however, astrocytes produce several pro-inflammatory factors that contribute to microglial activation. The interactions of microglia with neurons in the normal and diseased CNS are especially intriguing. Microglia are known to interact actively with neurons by facilitating axonal pruning during development, while neurons provide specific factors that alter microglial phenotypes and functions. This review focuses mainly on the roles of soluble neuronal factors that affect microglial phenotypes and functions and the possible involvement of these factors in the pathology of neurodegenerative diseases.

Keywords: microglia, neurons, neuroinflammation, transcriptional regulation, microRNA 


\section{INTRODUCTION}

Microglia are central nervous system (CNS)-resident myeloid cells that originate from common erythro-myeloid progenitors (EMPs), which themselves originate from embryonic stem cells (ESCs) in the yolk. Microglia populate the brain during early fetal development (Kierdorf et al., 2013; Ponomarev et al., 2013). Later, microglia harbor the ability to self-renew locally and remain in the CNS microenvironment throughout life, where they comprise $10-15 \%$ of all cells in the brain and spinal cord. In this respect, microglia differ from resident macrophages in other tissues, which originate from hematopoietic stem cells (HSCs) (Li and Barres, 2018), although resident myeloid Langerhans cells in several tissues, such as the skin, represent a mixture of cells derived from ESCs and HSCs (Collin and Milne, 2016). Under pathological conditions such as irradiation or inflammation, HSC-derived myeloid cells may also enter the CNS and acquire the characteristics of ESC-derived microglia, thus leading to a mixed population of HSC- and ESC-derived CNSresident macrophages similar to that of dermal Langerhans cells (Ponomarev et al., 2013).

Currently, we understand that the phenotypes and functions of microglia are influenced strongly by the local CNS microenvironment. Even in a diseased CNS, microglia in unaffected areas exhibit a normal phenotype and are referred to as homeostatic microglia, whereas microglia proximal to the damaged area exhibit a distinct disease-associated phenotype (Dubbelaar et al., 2018). Under normal conditions, homeostatic microglia are maintained at appropriate densities in various regions of the brain, mostly via local self-renewal ( $\mathrm{Li}$ and Barres, 2018; Hammond et al., 2019). During disease, microglia in damaged areas often exhibit heterogeneous and mixed-activation phenotypes and may play a beneficial or detrimental role, depending on the area, extent, and nature of neuronal damage and the stage of the disease (Li and Barres, 2018; Szepesi et al., 2018; Hammond et al., 2019). Therefore, it is important to understand how the phenotypes and functions of microglia are determined by specific signals from other CNS-resident cells, such as astrocytes and neurons. This review discusses the nature of such signals and mechanisms of adaptation to the local microenvironment.

\section{EPIGENETIC CHANGES MEDIATE ADAPTATION OF THE MICROGLIA TO TISSUE-SPECIFIC MICROENVIRONMENTS}

Modern studies have demonstrated that the adaptation of macrophages to local tissue microenvironments is mediated by epigenetic changes in chromatin (i.e., chromatin remodeling). This process enables the binding of several key transcription factors (TFs) that mediate microglial differentiation (Gosselin et al., 2017; Holtman et al., 2017). Like other tissue-resident macrophages, microglia reside in the CNS under the influence of tissue-derived factors that drive the expression of lineage-specific
TFs such as PU.1 and CEBP $\alpha$ (Gosselin et al., 2014). Both CEBP $\alpha$ and PU. 1 are induced by macrophage colony-stimulating factor-1 (CSF-1) or its analog, interleukin (IL)-34 (Ponomarev et al., 2013), which are produced by astrocytes and neurons (Frei et al., 1992; Mizuno et al., 2011). These factors drive the survival, renewal, and lineage-specific differentiation of microglia (Li and Barres, 2017). In addition to lineage-specific TFs, tissuespecific TFs complement and modulate the functions of core lineage-specific factors (Gosselin et al., 2014; Holtman et al., 2017), including SMAD family TFs driven by transforming growth factor (TGF) $\beta$, which is produced by astrocytes and neurons (Dobolyi et al., 2012). We found that mature microglia very strongly express TGF $\beta 1 \mathrm{mRNA}$ (unpublished observation), indicating that self-TGF $\beta 1$ could maintain SMAD2/3 expression in an autocrine manner. Several other factors, including but not limited to V-Maf musculoaponeurotic fibrosarcoma oncogene homolog B (MAFB), interferon response factor (IRF)8, Sallike (SALL)1, and peroxisome proliferator-activated receptor $(\mathrm{PPAR}) \gamma$, contribute to the transcriptional control of microglia identity in the normal CNS (Gosselin et al., 2014, 2017; Holtman et al., 2017). Under inflammatory conditions, other inducible TFs, such as nuclear factor (NF)ผB, NF-of activated T cells (AT), and signal transducer and activator of transcription (STAT) $1 / 3$, contribute further to the phenotypes of activated microglia (Holtman et al., 2017). The cooperative binding of various lineage- and tissue-specific TFs, such as PU.1, CEBP $\alpha$, IRF8, SMAD2/3, and SALL1, induce the modification of chromatin and generation of primed enhancers for gene expression, which is characterized by monomethylated $\mathrm{H} 3 \mathrm{~K} 4$ ( $\mathrm{H} 3 \mathrm{~K} 4 \mathrm{me} 1)$. In the diseased CNS, inflammatory stimuli [e.g., IL-6, tumor necrosis factor (TNF)] induces the binding of additional factors, such as NFKB, NF-AT, and STAT1/3, and the acetylation of H3K27 (H3K27Ac) (Holtman et al., 2017). Thus, the actions of multiple TFs induce epigenetic changes that lead to the remodeling of chromatin and the formation of a specific mature microglial phenotype. The exact mechanism underlying this highly complex process remains elusive.

\section{EPIGENETIC CHANGES IN THE ACTIVATION AND POLARIZATION OF MACROPHAGES AND MICROGLIA}

The phenotypes and functions of immune cells such as microglia and macrophages depend on the type of activation. One example is the existence of pro-inflammatory M1-like macrophages, which are activated by interferon (IFN) $\gamma$ and/or lipopolysaccharide (LPS), and M2-like macrophages, which are activated by IL- 4 and/or IL-13 and associated with the resolution of inflammation and repair of damaged tissues (Sica and Mantovani, 2012; Ley, 2017). The phenotypes of M1 and M2 macrophages are plastic, and the existing model of the activation and polarization spectrum oversimplifies the M1M2 phenotypic dichotomy, especially in vivo (Martinez and Gordon, 2014; Ransohoff, 2016). Nevertheless, in vitro studies have clearly delineated a distinct pattern of specific TF expression in M1 macrophages, which is characterized by STAT1, STAT2, 
IRF4, and NFKB. In contrast, the M2 macrophage phenotype is mediated by STAT6, GATA3, and PPAR $\gamma$ (Lawrence and Natoli, 2011; Cherry et al., 2014; Li et al., 2018). Regarding specific markers, M1 macrophages express nitric oxide (NO) produced by the enzyme NOS2, high levels of major histocompatibility (MHC) class II and CD86 receptors, and the cytokines TNF and IL-6. In contrast, M2 macrophages express arginase (Arg1), extracellular matrix-binding lectin (Ym1/Chi313), and the chemokine CCL22, a heparin-binding protein (Lawrence and Natoli, 2011; Krzyszczyk et al., 2018).

Histone methylation and acetylation are associated with both the M1 and M2 states of activated macrophages and microglia (Cheray and Joseph, 2018; Groot and de Pienta, 2018). The methylation of H4R3 has been shown to positively regulate M2 by inducing the expression of the M2-associated TF PPAR $\gamma$ in mouse peritoneal macrophages (PMs) activated with IL-4 (Tikhanovich et al., 2017). H3K4 methylation also positively regulates $\mathrm{M} 2$ polarization in human macrophages activated with M-CSF and IL-4 (Kittan et al., 2013). The enzyme JMJD3, known also as H3K27 demethylase, has been shown to be critical to the upregulation of IRF4 and Arg1 in mouse bone marrow-derived macrophages (BMDMs) stimulated with IL-4 (Satoh et al., 2010). The expression of JMJD3 is also upregulated in IL-4-treated mouse microglia, leading to H3K27 demethylation (Tang et al., 2014). Moreover, the acetylation of H3K9 and H3K14 in the Tnf, Il6, Nos2, and H2Ab (MHC class II) promoter regions is a critical inducer of the expression of these M1-associated molecules in LPS-stimulated mouse microglia (Chauhan et al., 2015).

Similar to other tissues, the CNS produces IL-4 via neurons and possibly astrocytes. This cytokine contributes to the expression of M2-like markers and may also induce the expression of the M2-associated TF PPAR $\gamma$ in mature microglia (Veremeyko et al., 2015, 2018b; Zhao et al., 2015). We found that normal microglia express Ym1 in a CNS-derived IL-4-dependent manner (Ponomarev et al., 2007). More recent studies based on single-cell RNA sequencing demonstrated that mouse microglia express Arg1, especially during the embryonic and early postnatal stages (Hammond et al., 2019). However, the initial concept of microglial polarization was criticized recently, as microglia were shown to express M1 and M2 markers simultaneously at the single-cell level (Ransohoff, 2016). We found this unsurprising, as we discovered long ago that microglia exhibited dual activation and an M1/M2 mixed phenotype in the context of experimental autoimmune encephalitis (Ponomarev et al., 2007). We believe that criticism of the concept of microglial polarization was mainly due to the misconception that microglia must exist in one of two mutually exclusive states (M1 or M2). More recent studies of macrophages and microglia demonstrated that these activation states are impermanent and somewhat dynamic. We also demonstrated that IL-4-activated macrophages exhibit a high level of plasticity, and that this is due to a high level of Egr2, which enables the cells to react to other activation stimuli (Veremeyko et al., 2015). Interestingly, mouse and human microglia express multiple early response genes, including Egr2, even at a single-cell level, which suggests a high level of cellular plasticity (Masuda et al., 2019). Importantly, therefore, microglia are exposed to a milieu of different cytokines (e.g., IL-4, TNF) during inflammation, and the state of activation represents a dynamic superposition of different activation pathways, rather than the mutual exclusion of the M1 and M2 states.

\section{ROLE OF MICRORNA IN MICROGLIAL PHENOTYPES AND FUNCTIONS}

In addition to histone methylation and acetylation, other epigenetic mechanisms regulate gene expression in microglia. One well-known mechanism involves the suppressive actions of regulatory microRNAs (miRNAs) against gene expression. MiRNAs are short (22-23 nucleotides) non-coding RNAs that bind complementarily to the mRNAs of target genes and either repress the translation or induce the degradation of these mRNAs (Ponomarev et al., 2013). In both cases, translation of the target gene and protein expression are decreased. The roles of miRNAs in microglial functions were reviewed recently (Guo et al., 2019). Moreover, a recent study demonstrated the microglial-neuronal transfer of exosome-associated miRNAs. M1-activated microglia were shown to produce extracellular vesicles containing the microglia-specific miRNA miR-146a-5p, which is not detected in neurons. Upon transfer, this miRNA downregulated the expression of the genes encoding presynaptic synaptotagmin-1 and postsynaptic neuroligin-1 (Prada et al., 2018). In this review, we focus on the roles of two neuronal miRNAs, miR-124 and miR-9, which are transferred from neurons to microglia and/or macrophages. Our screening of microRNAs that are expressed in microglia but not in resident PMs or BMDMs revealed the strong expression of miR-124 in the microglia (Ponomarev et al., 2011). Interestingly, miR-124 is expressed strongly in neurons and is responsible for the differentiation of neuronal progenitor cells (NPCs) into mature neurons (Ponomarev et al., 2013). We determined that miR-124 targeted $\operatorname{CEBP} \alpha$, a critical TF for macrophage and microglial differentiation. CEBP $\alpha$ was expressed in mouse embryonic (until E14) and early postnatal microglia (E15-P14) but not in mature microglia (P60), whereas miR124 was expressed strongly in mature microglia, but not in embryonic and early postnatal microglia. The transfection of BMDMs with miR-124 decreased the expression of CEBP $\alpha$ and PU.1 and the pro-inflammatory cytokines TNF and IL-6, and increased the expression of the anti-inflammatory factors Arg1, Fizz1, and TGF $\beta 1$ (Ponomarev et al., 2011). Indeed, exposure to the pro-inflammatory stimuli TNF and IL-6 increased the expression and DNA binding of $\mathrm{CEBP} \alpha$ and PU.1, which in turn increased the induction of downstream gene expression (Crotti et al., 2014). The upregulation of Arg1, Fizz1, and TGF $\beta 1$ in macrophages transfected with miR-124 was consistent with the phenotypes of M2-like macrophages activated with IL-4 or IL-13 (Ponomarev et al., 2011). Indeed, we found that IL-4 or IL-13 induced the expression of longer pre-miR124 transcripts in BMDMs and lung resident macrophages in a STAT6-dependent manner, whereas this process was not observed in mature microglia from a normal CNS (Veremeyko et al., 2013). Therefore, the microglial expression of neuronal miR-124 was upregulated via mechanisms other than the intrinsic transcription of longer miR-124 precursors and subsequent 
processing and maturation into functional miR-124. Further experiments indicated the horizontal transfer of miR-124 and miR-9 from neurons to microglia (Veremeyko et al., 2018a). Similar to miR-124, miR-9 is expressed strongly in neurons. This miRNA deactivates macrophages and microglia by targeting $\mathrm{NF} \mathrm{B}$, the expression of which is induced in microglia by inflammatory stimuli (Veremeyko et al., 2018a). Therefore, both miR-124 and miR-9 promote an anti-inflammatory phenotype in the normal CNS. These findings also indicate that normal neurons send continuous signals to microglia to maintain the latter cells in a quiescent state.

\section{HORIZONTAL TRANSFER OF MIRNA FROM NEURONS TO MICROGLIA}

Our understanding of the induction of miR-124 expression in mature microglia led us to hypothesize that this miRNA is transferred from mature neurons to microglia. Although previous reports described the transfer of miRNA between cell types (Barteneva et al., 2013, 2017), most demonstrated the transfer of miRNAs via exosomes or microparticles (Zhang et al., 2015). We investigated in detail the presence of the neuronally abundant microRNAs, miR-124 and miR-9, in the extracellular space. Notably, both miR-124 and miR-9 were secreted actively by electrically active neurons, which was confirmed by using tetrodotoxin (TTX) to block secretion (Veremeyko et al., 2018a). However, both miRNAs were secreted in various forms, including naked single-strand RNA (ssRNA), naked double-strand RNA (dsRNA), and ssRNA or dsRNA complexed with proteins, and within exosomes. Importantly, some proportion of miR-9 was co-localized with exosomes, whereas miR-124 was not associated with exosomes. Rather, miR-124 formed complexes with high-density lipoproteins (HDLs), which are produced in the CNS by astrocytes. These HDL-miR-124 complexes translocated efficiently into the cytoplasm of microglia and macrophages via scavenger receptors. This finding highlights an important mechanism by which functional neurons send continuous signals to the microglia to provide instruction and maintain a resting (nonactivated) phenotype in the normal CNS (Veremeyko et al., 2018a). We propose that neuronal electric and synaptic activity decreases, miR-124 and miR-9 secretions are blocked, and the microglial phenotype shifts from resting to activated during neurodegeneration.

\section{ANALOGOUS REGULATION OF GENE EXPRESSION IN MICROGLIA AND NEURONS}

We hypothesized that gene expression is regulated similarly, in neurons and microglia, as both cell types are closely associated with each other and exposed to the same microenvironment. Several findings support our hypothesis. First, we observed that microglia express neuronal miRNAs such as miR-124 and miR-9 (Veremeyko et al., 2018a). Second, microglia produce neuronal trophic factors such as brain-derived neurotrophic factor (BDNF) (Parkhurst et al., 2013). Third, microglia express Egr1, c-Jun, and c-Fos, which are products of neuronal early response genes (Holtman et al., 2017). Fourth, microglia express Sall1, which, together with Egr1, is associated with neuronal maturation and synaptic pruning (Buttgereit et al., 2016). Egr1, c-Jun, and c-Fos are hallmarks of neuronal synaptic activity (Dukhinova et al., 2018), while Egr2 is involved in macrophage activation, polarization, and plasticity (Veremeyko et al., 2015). However, c-Fos forms complexes with c-Jun to form activator protein-1 (AP-1), a very potent TF associated with macrophage activation (Hop et al., 2018). AP-1 induces the expression of proinflammatory genes in macrophages and microglia (MatcovitchNatan et al., 2016; Holtman et al., 2017). Alone, c-Fos suppresses the expression of M1-associated genes such as Nos2, Tnf, and Il6 (Okada, 2003; Ray et al., 2006). CEBP $\beta$ is another interesting TF that is regulated in macrophages by Egr1-3 and the cAMP pathway (Veremeyko et al., 2015, 2018b). CEBP $\beta$ is important for cortical neurogenesis and may thus induce the expression of both myeloid and neuronal genes (Ménard et al., 2002). Sall1 also regulates cortical neurogenesis (Harrison et al., 2012) and serves as a unique marker of microglia. The miRNA miR-124 inactivates the transcriptional repressor REST, which in turn inhibits the expression of neuronal genes in non-neuronal cell types (Visvanathan et al., 2007). Mir-124 is expressed in microglia and M2 macrophages. Sall1-deficient or miR-124 inhibitor-treated microglia lose their processes that are typical for adult microglia in normal CNS (ramified form) and take a more activated amoeboid form, indicating that the resting microglial phenotype might be maintained by neuronal genes (Ponomarev et al., 2011; Koso et al., 2016, 2018). In other words, neurons and mature microglia share similar expression patterns for several genes. We believe that these neuronal genes may contribute to the unique phenotypes of microglia in the CNS. However, the induction of neuronal gene expression in microglia remains unclear and must be addressed. We propose that neuronal gene expression in microglia is associated with similar epigenetic changes that occur in both cell types during development. During neural lineage commitment, $\mathrm{H} 3 \mathrm{~K} 27$ in the promoter regions of many neural lineage genes is demethylated by the enzyme JMJD3 (Choi et al., 2015), similar to the effects of IL-4 on mouse BMDMs. Simultaneously, H3K27 is methylated in non-neuronal cells (including nonactivated peripheral macrophages) (Satoh et al., 2010). These findings indicate similar epigenetic changes in microglia, M2 macrophages, and neuronal cells.

Besides the demethylation of H3K27 in M2 macrophages, miR-124 is upregulated by the IL- 4 and cAMP pathways in mouse BMDM (Veremeyko et al., 2018b), while microglia receive horizontal transfers of miR-124 and miR-9 from neurons (Veremeyko et al., 2018a). Therefore, we propose that neuronal miR-124 and miR-9 inhibit REST in microglia, leading to the expression of several neuronal genes, similar to the consequences of miR-124 and miR-9 overexpression in fibroblasts (Lee et al., 2018). As the deactivation of REST and upregulation of miR124 were shown to be associated with the modification of the neuronal BAF chromatin remodeling complex during neuronal 
A

\section{ESC}

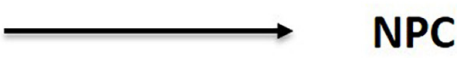

npBAF complex

esBAF complex

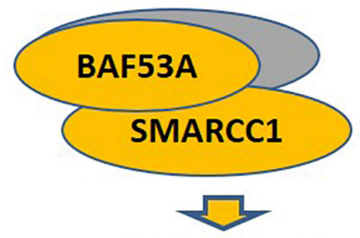

Pluripotent

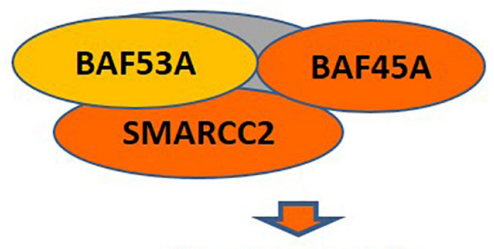

Neurogenesis

Neuron

nBAF complex

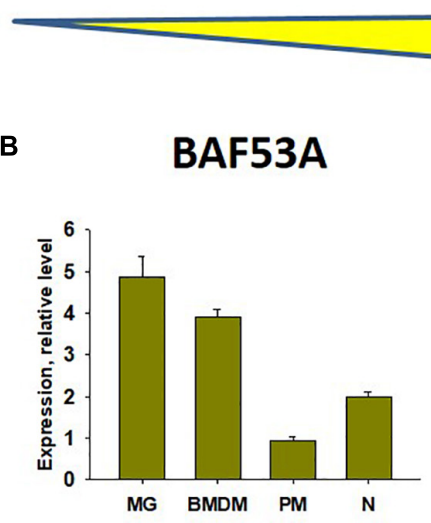

E

SMARCC1

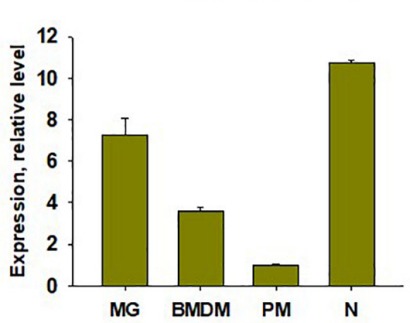

c

BAF45A

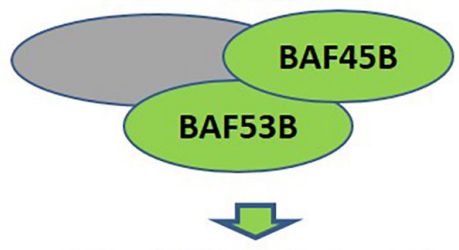

Dendritic Outgrowth

Expression of neuronal genes:

BEX, SYT1, MAP2, TUBB3

B

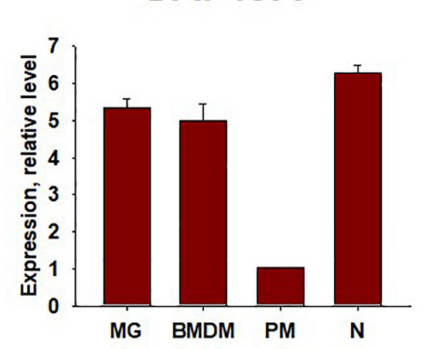

D

BAF53B

\section{F}

SMARCC2
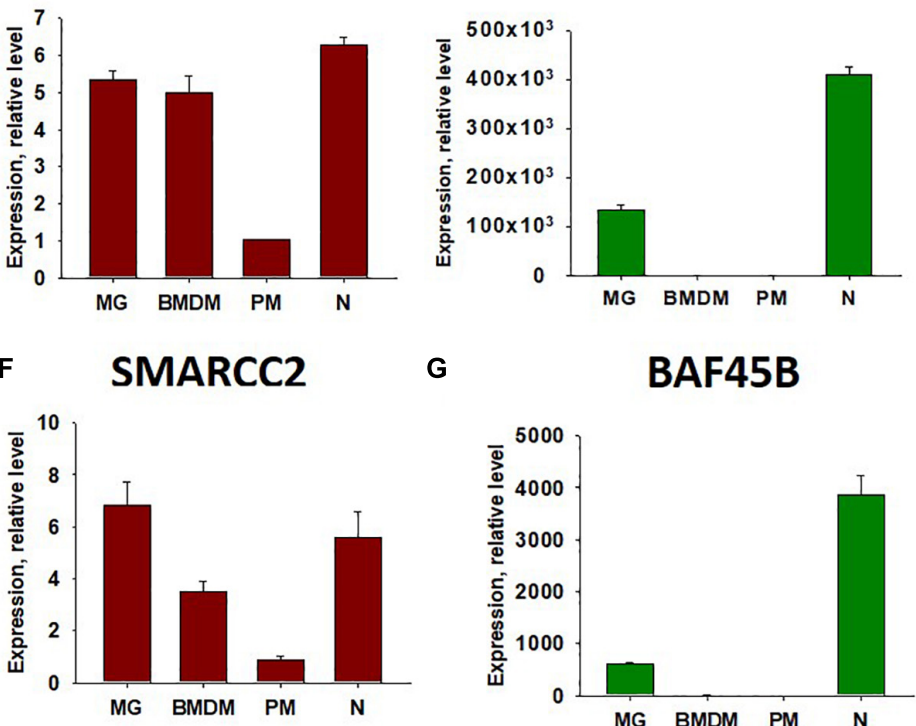

BAF45B

H

Neuronal Genes
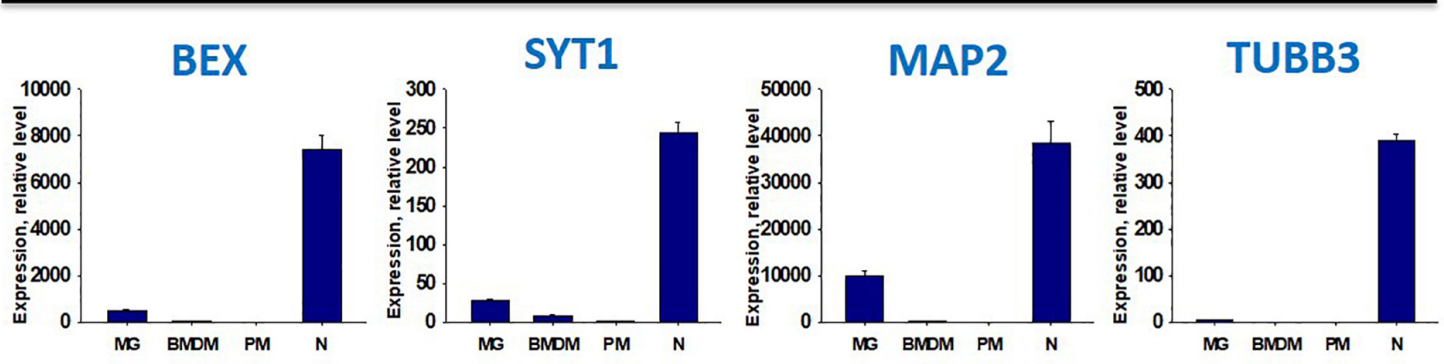

FIGURE 1 | Comparison of expression of factors of chromatin remodeling BAF complexes at the stage of embryonic stems cells (esBAF), neuronal progenitor cells (npBAF), and neurons (nBAF) as well as analysis of expression neuronal genes in microglia, bone marrow-derived macrophages, peritoneal macrophages, and cultured cortical neurons. (A) Schematic diagram of Brm/Brg-associated factor (BAF) chromatin remodeling complex for three stages of neuronal differentiation from embryonic stem cells (ESC) to neuronal progenitor cells (NPC) and to mature neurons (Neuron) (Adapted from "Epigenetic regulation of neural differentiation from embryonic stem cells" by Atsushi Shimomura and Eri Hashino with permission. 2013 Shimomura A., Hashino E. Published in Trends in Cell Signaling Pathways in Neuronal Fate Decision under CC BY 3.0 license. Available from: htpp://dx.doi.org/10.5772/53650). (B-G) Expression of factors of BAF complex BAF53A (B), BAF45A (C), BAF53B (D), SMARCC1 (E), SMARCC2 (F), and BAF45B (G) in microglia (MG), bone marrow-derived macrophages (BMDM), peritoneal macrophages $(P M)$, and cultured cortical neurons (N). (H) Expression of neuronal genes BEX, SYT1, MAP2, and TUBB3 in microglia (MG), bone marrow-derived macrophages (BMDF), peritoneal macrophages (PM), and cultured cortical neurons $(\mathrm{N})$. 
TABLE 1 | Neuron-derived soluble factors that affect the activation state of microglia in normal CNS and during neuroinflammation and neurodegeneration.

\begin{tabular}{|c|c|c|c|}
\hline Class of mediators & Neuron-derived mediators & $\begin{array}{l}\text { Changes during } \\
\text { neuroinflammation/ } \\
\text { neurodegeneration }\end{array}$ & Effect on microglia \\
\hline \multirow[t]{3}{*}{ Cytokines } & $\mathrm{IL}-4$ & Decreased & $\begin{array}{l}\text { Promote anti-inflammatory phenotype and expression of: Ym1, Fizz1, } \\
\text { TGF } \beta 1 \text { (Veremeyko et al., 2015, 2018b; Zhao et al., 2015; Cheray and } \\
\text { Joseph, 2018) }\end{array}$ \\
\hline & TGF $\beta 1$ & Decreased & $\begin{array}{l}\text { Promote homeostatic phenotype of microglia in normal adult CNS (Dobolyi } \\
\text { et al., 2012) }\end{array}$ \\
\hline & $\mathrm{IL}-34$ & Increased & $\begin{array}{l}\text { Homeostatic maintenance, proliferation, and survival of microglia (Frei et al., } \\
\text { 1992; Mizuno et al., 2011; Ponomarev et al., 2013) }\end{array}$ \\
\hline \multirow[t]{3}{*}{ Chemokines } & CCL2 & Increased & Microglia migration, activation, and proliferation (Haas et al., 2019) \\
\hline & CXCL12 & Increased & Microglia migration and activation (Haas et al., 2019) \\
\hline & CX3CL1 (soluble form) & Decreased & Deactivate microglia (Haas et al., 2019) \\
\hline \multirow[t]{2}{*}{ Growth Factors } & NGF & Increased & Promote anti-inflammatory phenotype of microglia (Fodelianaki et al., 2019) \\
\hline & BDNF & Increased & $\begin{array}{l}\text { Suppress release of NO in microglia. Promote anti-inflammatory phenotype } \\
\text { (Lai et al., 2018) }\end{array}$ \\
\hline \multirow[t]{4}{*}{ Neurotransmitters } & Serotonin $(5 \mathrm{HT})$ & $\begin{array}{l}\text { Decreased during } \\
\text { TBl-induced } \\
\text { neuroinflammation }\end{array}$ & $\begin{array}{l}\text { Decreased IFN } \gamma \text {-induced activation of macrophages and possibly microglia } \\
\text { (Liu et al., 2016) }\end{array}$ \\
\hline & Dopamine & Decreased & Possibly suppress microglia activation via DR2 (Liu et al., 2016) \\
\hline & Glutamate & Decreased & $\begin{array}{l}\text { Promote microglia activation and expression of TNF and NO (Liu et al., } \\
\text { 2016) }\end{array}$ \\
\hline & GABA & Decreased & $\begin{array}{l}\text { Inhibit microglia activation and release LPS-induced TNF and IL-6 (Liu et al., } \\
\text { 2016) }\end{array}$ \\
\hline $\begin{array}{l}\text { Low-molecular-weight } \\
\text { mediators }\end{array}$ & ATP & Increased & Activate microglia (Davalos et al., 2005) \\
\hline microRNAs & miR-124 miR-9 & $\begin{array}{l}\text { Decreased } \\
\text { Decreased }\end{array}$ & Deactivate microglia (Veremeyko et al., 2018a) \\
\hline \multirow[t]{2}{*}{ Complement } & C3 & Increased & Mediate synaptic pruning by microglia (Luchena et al., 2018) \\
\hline & C1q & Increased & \\
\hline
\end{tabular}

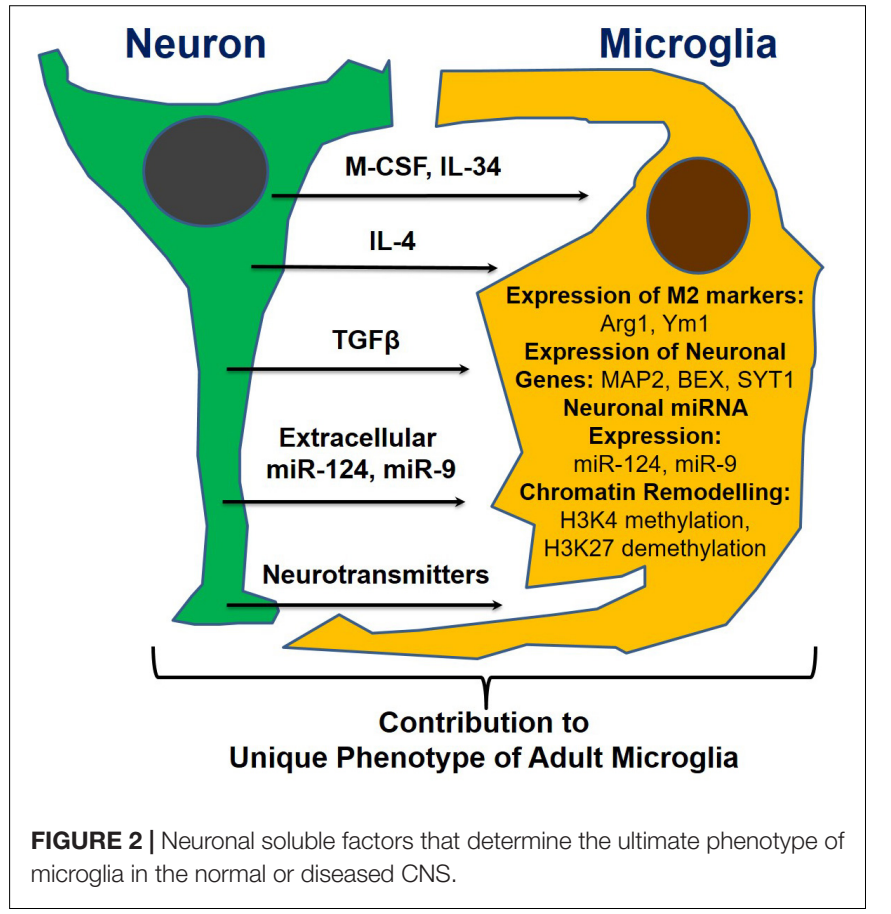

lineage commitment (Choi et al., 2015), we hypothesized that BAF would also be modified in the microglia, compared with non-activated BMDM. To test this hypothesis, we investigated the neuronal BAF chromatin remodeling complex (Choi et al., 2015), which regulates the expression of neuronal genes during differentiation from embryonic stem cells (ESCs) to neuronal progenitor cells (NPCs) from NPCs to mature neurons (Shimomura and Hashino, 2013), in neurons, peritoneal macrophages (PMs), BMDMs, and microglia (Figure 1A). In the ESC stage, the esBAF complex exists, and the co-factors $B A F 53 A$ and SMARCC1 are expressed predominantly. In the NPC stage, the npBAF complex retains $B A F 53 A$ but includes two new factors, BAF45A and SMARCC2. Finally, in mature neurons, the nBAF complex comprises $B A F 45 B$ and $B A F 53 B$. The expression of the neuronal genes $B E X, S Y T 1, M A P 2$, and TUBB3 increased gradually during the $\mathrm{ESC} \rightarrow \mathrm{NPC} \rightarrow$ neuron transition path (Figure 1A). We similarly compared the expression levels of transcripts encoding factors in the esBAF, npBAF, and $\mathrm{nBAF}$ complexes in mature microglia, BMDMs, PMs, and cultured mature cortical neurons, as described in our earlier studies (Veremeyko et al., 2015, 2018a; Dukhinova et al., 2018, 2019). We observed a similar expression of BAF53A and SMARCC1, which encode esBAF components, between microglia and neurons and 
between microglia and BMDMs. However, these genes were expressed at significantly higher levels in microglia than in PMs (Figures 1B,E). We observed a similar pattern in the expression of BAF45A and SMARCC2, which encode npBAF components. These genes were expressed similarly in microglia and neurons, at similar or slightly higher levels in microglia relative to BMDMs, and at significantly higher levels in microglia than in PMs (Figures 1C,F). However, BAF53B and BAF45B, which encode $\mathrm{nBAF}$ components, were only expressed at detectable levels in neurons and microglia, but not BMDMs and PMs (Figures 1D,G). The neuronal genes BEX, SYT1, and MAP2 were expressed in neurons and microglia, but not in BMDMs and PMs (Figure 1H). TUBB3, the most abundant neuronal gene, was not expressed in microglia, indicating that the previous results could not be attributed to the contamination of microglia preparations with neuronal cells (Figure 1H, TUBB3). Therefore, in contrast with other macrophages, microglia express factors associated with the nBAF complex, as well as several neuronal genes. Although the roles of neuronal gene products such as MAP2 in the microglial phenotype remain unclear, we propose that these factors contribute to the unique phenotype of mature microglia in the normal CNS.

\section{ROLES OF NEURONAL SOLUBLE FACTORS IN THE MAINTENANCE OF THE MICROGLIAL PHENOTYPE}

Following our discovery of the horizontal transfer of neuronal miR-9 and miR-124 from electrically active neurons to microglia, we hypothesized that the microglial phenotype is influenced strongly by soluble neuronal factors (Table $\mathbf{1}$ and Figure 2). Accordingly, these soluble neuronal factors exhibit great potential for future therapeutic interventions targeting neurodegenerative diseases. Both astrocytes and neurons can produce IL-4, TGF $\beta 1$, and IL-34, which greatly affect the microglial phenotype, as discussed previously (Table 1). Neuronal IL-4 might further contribute to $\mathrm{H} 3 \mathrm{~K} 27$ demethylation in microglia (Cheray and Joseph, 2018). Neurons also produce the chemokines CCL2, CCL12, and CX3CL1 (both membrane-bound and soluble forms), which can induce microglial migration toward neurons (CCL2 and CCL12) and microglial deactivation (CX3CL1) (Haas et al., 2019). Neuronal growth factor (NGF) and BDNF also contribute to the anti-inflammatory microglial phenotype (Table 1; Lai et al., 2018; Fodelianaki et al., 2019). Neurotransmitters are other important molecules released by electrically active neurons that can directly affect microglia. Common neurotransmitters, such as serotonin, dopamine, and GABA, deactivate microglia, while glutamate activates these cells (Table 1; Liu et al., 2016). ATP is released by activated and

\section{REFERENCES}

Barteneva, N. S., Baiken, Y., Fasler-Kan, E., Alibek, K., Wang, S., Maltsev, N., et al. (2017). Extracellular vesicles in gastrointestinal cancer in conjunction with microbiota: on the border of kingdoms. Biochim. damaged neurons and contributes to microglial activation and inflammation (Davalos et al., 2005). The neuronal microRNAs miR-124 and miR-9 are transferred to the microglial cytoplasm, where they downregulate the expression of CEBP $\alpha$ and $\mathrm{NF} \kappa \mathrm{B}$ and deactivate the cells (Table 1; Veremeyko et al., 2018a). Finally, the complement components $\mathrm{C} 1 \mathrm{q}$ and $\mathrm{C} 3$ mediate neuronal axonal pruning by microglia via complement receptors (Table 1). Interestingly, C1q expression is induced in neurons via a TGF $\beta 1$ mediated mechanism, while C3 is present in neuronal synapses (Luchena et al., 2018). In summary, many factors, including proand anti-inflammatory stimuli, affect communication between neurons and microglia. The balance of these stimuli determines the ultimate phenotype of microglia in the normal or diseased CNS (Figure 2).

\section{CONCLUDING REMARKS}

Microglia represent a unique population of resident myeloid cells in the CNS. The phenotypes of these cells are influenced strongly by the local microenvironment and determined by the specific regulation of gene expression at the epigenetic level. Currently, neuronal cells are thought to actively influence the phenotypes of microglia. In the normal CNS, electrically and synaptically active neurons maintain microglia in a deactivated state, whereas changes in the balance of inhibitory vs. activating stimuli in the contexts of neuroinflammatory and neurodegenerative diseases cause immediate changes in the microglial phenotype and induce activation. A further understanding of the interactions of neurons and microglia in the normal CNS would ultimately lead to the discovery of new pathways. This information would suggest potential mechanisms by which homeostasis could be restored in pathological settings, as well as potential treatment options for neurodegenerative diseases such as multiple sclerosis, Alzheimer's disease, and Parkinson's disease.

\section{AUTHOR CONTRIBUTIONS}

TV and EP conceived the review and study. TV, AY, and MD performed the experiments. TV, TS, and EP analyzed the data. TS and EP prepared the manuscript.

\section{FUNDING}

This work was supported by Research Grant CouncilGeneral Research Fund, reference no. 14113316 (Hong Kong Government, Hong Kong), and by Research Grant CouncilAreas of Excellence Fund grant (Hong Kong Government, Hong Kong), reference no. AoE/M-604/16.

Biophys. Acta Rev. Cancer 1868, 372-393. doi: 10.1016/j.bbcan.2017. 06.005

Barteneva, N. S., Fasler-Kan, E., Bernimoulin, M., Stern, J. N. H., Ponomarev, E. D., Duckett, L., et al. (2013). Circulating microparticles: square the circle. BMC Cell Biol. 14:23. doi: 10.1186/1471-2121-14-23 
Buttgereit, A., Lelios, I., Yu, X., Vrohlings, M., Krakoski, N. R., Gautier, E. L., et al. (2016). Sall1 is a transcriptional regulator defining microglia identity and function. Nat. Immunol. 17, 1397-1406. doi: 10.1038/ni.3585

Chauhan, A., Quenum, F. Z., Abbas, A., Bradley, D. S., Nechaev, S., Singh, B. B., et al. (2015). Epigenetic modulation of microglial inflammatory gene loci in helminth-induced immune suppression: implications for immune regulation in neurocysticercosis. ASN Neuro 7:1759091415592126. doi: $10.1177 /$ 1759091415592126

Cheray, M., and Joseph, B. (2018). Epigenetics control microglia plasticity. Front. Cell Neurosci. 12:243. doi: 10.3389/FNCEL.2018.00243

Cherry, J. D., Olschowka, J. A., and O'Banion, M. K. (2014). Neuroinflammation and M2 microglia: the good, the bad, and the inflamed. J. Neuroinflammation 11:98. doi: 10.1186/1742-2094-11-98

Choi, K.-Y., Yoo, M., and Han, J.-H. (2015). Toward understanding the role of the neuron-specific BAF chromatin remodeling complex in memory formation. Exp. Mol. Med. 47, e155-e155. doi: 10.1038/emm.2014.129

Collin, M., and Milne, P. (2016). Langerhans cell origin and regulation. Curr. Opin. Hematol. 23, 28-35. doi: 10.1097/MOH.0000000000000202

Crotti, A., Benner, C., Kerman, B. E., Gosselin, D., Lagier-Tourenne, C., Zuccato, C., et al. (2014). Mutant huntingtin promotes autonomous microglia activation via myeloid lineage-determining factors. Nat. Neurosci. 17, 513-521. doi: 10. 1038/nn.3668

Davalos, D., Grutzendler, J., Yang, G., Kim, J. V., Zuo, Y., Jung, S., et al. (2005). ATP mediates rapid microglial response to local brain injury in vivo. Nat. Neurosci. 8, 752-758. doi: 10.1038/nn1472

Dobolyi, A., Vincze, C., Pál, G., and Lovas, G. (2012). The neuroprotective functions of transforming growth factor beta proteins. Int. J. Mol. Sci. 13, 8219-8258. doi: 10.3390/IJMS13078219

Dubbelaar, M. L., Kracht, L., and Eggen, B. J. L. (2018). Boddeke EWGM. The kaleidoscope of microglial phenotypes. Front. Immunol. 9:1753. doi: 10.3389/ fimmu.2018.01753

Dukhinova, M., Kuznetsova, I., Kopeikina, E., Veniaminova, E., Yung, A. W. Y. Y., Veremeyko, T., et al. (2018). Platelets mediate protective neuroinflammation and promote neuronal plasticity at the site of neuronal injury. Brain Behav. Immun. 74, 7-27. doi: 10.1016/j.bbi.2018.09.009

Dukhinova, M., Veremeyko, T., Yung, A. W. Y., Kuznetsova, I. S., Lau, T. Y. B., Kopeikina, E., et al. (2019). Fresh evidence for major brain gangliosides as a target for the treatment of Alzheimer's disease. Neurobiol. Aging 77, 128-143. doi: 10.1016/j.neurobiolaging.2019.01.020

Fodelianaki, G., Lansing, F., Bhattarai, P., Troullinaki, M., Zeballos, M. A., Charalampopoulos, I., et al. (2019). Nerve growth factor modulates LPSinduced microglial glycolysis and inflammatory responses. Exp. Cell Res. 377, 10-16. doi: 10.1016/J.YEXCR.2019.02.023

Frei, K., Nohava, K., Malipiero, U. V., Schwerdel, C., and Fontana, A. (1992). Production of macrophage colony-stimulating factor by astrocytes and brain macrophages. J. Neuroimmunol. 40, 189-195. doi: 10.1016/0165-5728(92) 90133-6

Gosselin, D., Link, V. M., Romanoski, C. E., Fonseca, G. J., Eichenfield, D. Z., Spann, N. J., et al. (2014). Environment drives selection and function of enhancers controlling tissue-specific macrophage identities. Cell 159, 13271340. doi: 10.1016/j.cell.2014.11.023

Gosselin, D., Skola, D., Coufal, N. G., Holtman, I. R., Schlachetzki, J. C. M., Sajti, E., et al. (2017). An environment-dependent transcriptional network specifies human microglia identity. Science 356:eaal3222. doi: 10.1126/science.aal3222

Groot, A. E., and de Pienta, K. J. (2018). Epigenetic control of macrophage polarization: implications for targeting tumor-associated macrophages. Oncotarget 9, 20908-20927. doi: 10.18632/ONCOTARGET.24556

Guo, Y., Hong, W., Wang, X., Zhang, P., Körner, H., Tu, J., et al. (2019). MicroRNAs in microglia: how do microRNAs affect activation, inflammation, polarization of microglia and mediate the interaction between microglia and glioma? Front. Mol. Neurosci. 12:125. doi: 10.3389/fnmol.2019.00125

Haas, A. H., de, Weering, H. R. J., van, Jong, E. K., de Boddeke, H. W. G. M., et al. (2019). Neuronal chemokines: versatile messengers in central nervous system cell interaction. Mol. Neurobiol. 36, 137-151. doi: 10.1007/S12035-007-0036-8

Hammond, T. R., Dufort, C., Dissing-Olesen, L., Giera, S., Young, A., Wysoker, A., et al. (2019). Single-cell RNA sequencing of microglia throughout the mouse lifespan and in the injured brain reveals complex cell-state changes. Immunity 50, 253.e6-271.e6. doi: 10.1016/j.immuni.2018.11.004
Harrison, S. J., Nishinakamura, R., Jones, K. R., and Monaghan, A. P. (2012). Sall1 regulates cortical neurogenesis and laminar fate specification in mice: implications for neural abnormalities in Townes-Brocks syndrome. Dis. Model Mech. 5, 351-365. doi: 10.1242/dmm.002873

Holtman, I. R., Skola, D., and Glass, C. K. (2017). Transcriptional control of microglia phenotypes in health and disease. J. Clin. Invest. 127, 3220-3229. doi: 10.1172/JCI90604

Hop, H. T., Arayan, L. T., Huy, T. X. N., Reyes, A. W. B., Vu, S. H., Min, W., et al. (2018). The key role of c-Fos for immune regulation and bacterial dissemination in brucella infected macrophage. Front. Cell Infect Microbiol. 8:287. doi: $10.3389 /$ fcimb.2018.00287

Kierdorf, K., Erny, D., Goldmann, T., Sander, V., Schulz, C., Perdiguero, E. G., et al. (2013). Microglia emerge from erythromyeloid precursors via Pu.1- and Irf 8-dependent pathways. Nat. Neurosci. 16, 273-280. doi: 10.1038/nn.3318

Kittan, N. A., Allen, R. M., Dhaliwal, A., Cavassani, K. A., Schaller, M., Gallagher, K. A., et al. (2013). Cytokine induced phenotypic and epigenetic signatures are key to establishing specific macrophage phenotypes. PLoS One 8:e78045. doi: 10.1371/journal.pone.0078045

Koso, H., Nishinakamura, R., and Watanabe, S. (2018). Sall1 regulates microglial morphology cell autonomously in the developing retina. Adv. Exp. Med. Biol. 1074, 209-215. doi: 10.1007/978-3-319-75402-4_26

Koso, H., Tsuhako, A., Lai, C.-Y., Baba, Y., Otsu, M., Ueno, K., et al. (2016). Conditional rod photoreceptor ablation reveals Sall1 as a microglial marker and regulator of microglial morphology in the retina. Glia 64, 2005-2024. doi: 10.1002/glia.23038

Krzyszczyk, P., Schloss, R., Palmer, A., and Berthiaume, F. (2018). The role of macrophages in acute and chronic wound healing and interventions to promote pro-wound healing phenotypes. Front. Physiol. 9:419. doi: 10.3389/fphys.2018. 00419

Lai, S.-W., Chen, J.-H., Lin, H.-Y., Liu, Y.-S., Tsai, C.-F., Chang, P.-C., et al. (2018). Regulatory effects of neuroinflammatory responses through brainderived neurotrophic factor signaling in microglial cells. Mol. Neurobiol. 55, 7487-7499. doi: 10.1007/s12035-018-0933-z

Lawrence, T., and Natoli, G. (2011). Transcriptional regulation of macrophage polarization: enabling diversity with identity. Nat. Rev. Immunol. 11, 750-761. doi: $10.1038 /$ nri3088

Lee, S. W., Oh, Y. M., Lu, Y.-L., Kim, W. K., and Yoo, A. S. (2018). MicroRNAs overcome cell fate barrier by reducing EZH2-controlled REST stability during neuronal conversion of human adult fibroblasts. Dev. Cell 46, 73.e7-84.e7. doi: 10.1016/j.devcel.2018.06.007

Ley, K. (2017). M1 means kill. M2 means heal. J. Immunol. 199, 2191-2193. doi: 10.4049/JIMMUNOL.1701135

Li, H., Jiang, T., Li, M.-Q., Zheng, X.-L., and Zhao, G.-J. (2018). Transcriptional regulation of macrophages polarization by microRNAs. Front. Immunol. 9:1175. doi: 10.3389/fimmu.2018.01175

Li, Q., and Barres, B. A. (2017). Microglia and macrophages in brain homeostasis and disease. Nat. Rev. Immunol. 18, 225-242. doi: 10.1038/nri.2017.125

Li, Q., and Barres, B. A. (2018). Microglia and macrophages in brain homeostasis and disease. Nat. Rev. Immunol. 18, 225-242. doi: 10.1038/nri.2017.125

Liu, H., Leak, R. K., and Hu, X. (2016). Neurotransmitter receptors on microglia. Stroke Vasc Neurol. 1, 52-58. doi: 10.1136/svn-2016-000012

Luchena, C., Zuazo-Ibarra, J., Alberdi, E., Matute, C., and Capetillo-Zarate, E. (2018). Contribution of neurons and glial cells to complement-mediated synapse removal during development, aging and in Alzheimer's disease. Mediators Inflamm. 2018, 1-12. doi: 10.1155/2018/2530414

Martinez, F. O., and Gordon, S. (2014). The M1 and M2 paradigm of macrophage activation: time for reassessment. F1000Prime Rep. 6:13. doi: 10.12703/P6-13

Masuda, T., Sankowski, R., Staszewski, O., Böttcher, C., Amann, L., Sagar, et al. (2019). Spatial and temporal heterogeneity of mouse and human microglia at single-cell resolution. Nature 566, 388-392. doi: 10.1038/s41586-0190924-x

Matcovitch-Natan, O., Winter, D. R., Giladi, A., Vargas Aguilar, S., Spinrad, A., Sarrazin, S., et al. (2016). Microglia development follows a stepwise program to regulate brain homeostasis. Science 353, aad8670. doi: 10.1126/science.aad8670

Ménard, C., Hein, P., Paquin, A., Savelson, A., Yang, X. M., Lederfein, D., et al. (2002). An essential role for a MEK-C/EBP pathway during growth factorregulated cortical neurogenesis. Neuron 36, 597-610. doi: 10.1016/S08966273(02)01026-7 
Mizuno, T., Doi, Y., Mizoguchi, H., Jin, S., Noda, M., Sonobe, Y., et al. (2011). Interleukin-34 selectively enhances the neuroprotective effects of microglia to attenuate oligomeric amyloid- $\beta$ neurotoxicity. Am. J. Pathol. 179, 2016-2027. doi: 10.1016/j.ajpath.2011.06.011

Okada, S. (2003). Dominant-negative effect of the c-fos family gene products on inducible NO synthase expression in macrophages. Int. Immunol. 15, 12751282. doi: $10.1093 /$ intimm/dxg126

Parkhurst, C. N., Yang, G., Ninan, I., Savas, J. N., Yates, J. R., Lafaille, J. J., et al. (2013). Microglia promote learning-dependent synapse formation through brain-derived neurotrophic factor. Cell 155, 1596-1609. doi: 10.1016/j.cell.2013. 11.030

Ponomarev, E. D., Maresz, K., Tan, Y., and Dittel, B. N. (2007). CNS-derived interleukin-4 is essential for the regulation of autoimmune inflammation and induces a state of alternative activation in microglial cells. J. Neurosci. 27, 10714-10721. doi: 10.1523/JNEUROSCI.1922-07.2007

Ponomarev, E. D., Veremeyko, T., Barteneva, N., Krichevsky, A. M., and Weiner, H. L. (2011). MicroRNA-124 promotes microglia quiescence and suppresses EAE by deactivating macrophages via the C/EBP- $\alpha$-PU.1 pathway. Nat. Med. 17, 64-70. doi: 10.1038/nm.2266

Ponomarev, E. D., Veremeyko, T., and Weiner, H. L. (2013). MicroRNAs are universal regulators of differentiation, activation, and polarization of microglia and macrophages in normal and diseased CNS. Glia 61, 91-103. doi: 10.1002/ glia. 22363

Prada, I., Gabrielli, M., Turola, E., Iorio, A., D’Arrigo, G., Parolisi, R., et al. (2018). Glia-to-neuron transfer of miRNAs via extracellular vesicles: a new mechanism underlying inflammation-induced synaptic alterations. Acta Neuropathol. 135, 529-550. doi: 10.1007/s00401-017-1803-x

Ransohoff, R. M. (2016). A polarizing question: do M1 and M2 microglia exist. Nat. Neurosci. 19, 987-991. doi: 10.1038/nn.4338

Ray, N., Kuwahara, M., Takada, Y., Maruyama, K., Kawaguchi, T., Tsubone, H., et al. (2006). c-Fos suppresses systemic inflammatory response to endotoxin. Int. Immunol. 18, 671-677. doi: 10.1093/intimm/dxl004

Satoh, T., Takeuchi, O., Vandenbon, A., Yasuda, K., Tanaka, Y., Kumagai, Y., et al. (2010). The Jmjd3-Irf4 axis regulates M2 macrophage polarization and host responses against helminth infection. Nat. Immunol. 11, 936-944. doi: 10.1038/ ni. 1920

Shimomura, A., and Hashino, E. (2013). "Epigenetic regulation of neural differentiation from embryonic stem cells," in Trends in Cell Signaling Pathways in Neuronal Fate Decision, ed. S. Wislet (London: IntechOpen Limited), 305325.

Sica, A., and Mantovani, A. (2012). Macrophage plasticity and polarization: in vivo veritas. J. Clin. Invest 122, 787-795. doi: 10.1172/JCI59643

Szepesi, Z., Manouchehrian, O., Bachiller, S., and Deierborg, T. (2018). Bidirectional microglia-neuron communication in health and disease. Front. Cell Neurosci. 12:323. doi: 10.3389/fncel.2018.00323

Tang, Y., Li, T., Li, J., Yang, J., Liu, H., Zhang, X. J., et al. (2014). Jmjd3 is essential for the epigenetic modulation of microglia phenotypes in the immune pathogenesis of Parkinson's disease. Cell Death. Differ. 21, 369-380. doi: 10.1038/cdd. 2013.159

Tikhanovich, I., Zhao, J., Olson, J., Adams, A., Taylor, R., Bridges, B., et al. (2017). Protein arginine methyltransferase 1 modulates innate immune responses through regulation of peroxisome proliferator-activated receptor $\gamma$-dependent macrophage differentiation. J. Biol. Chem. 292, 6882-6894. doi: 10.1074/JBC. M117.778761

Veremeyko, T., Kuznetsova, I. S., Dukhinova, M., Yung, A. W. Y., Kopeikina, E., Barteneva, N. S. P. E., et al. (2018a). Neuronal extracellular microRNAs miR-124 and miR-9 mediate cell-cell communication between neurons and microglia. J. Neurosci. Res. 97, 162-184. doi: 10.1002/jnr.24344

Veremeyko, T., Yung, A. W. Y., Dukhinova, M., Kuznetsova, I. S., Pomytkin, I., Lyundup, A., et al. (2018b). Cyclic AMP pathway suppress autoimmune neuroinflammation by inhibiting functions of encephalitogenic CD4 T cells and enhancing M2 macrophage polarization at the site of inflammation. Front. Immunol. 9:50. doi: 10.3389/fimmu.2018.00050

Veremeyko, T., Siddiqui, S., Sotnikov, I., Yung, A., and Ponomarev, E. D. (2013). IL-4/IL-13-dependent and independent expression of miR-124 and its contribution to M2 phenotype of monocytic cells in normal conditions and during allergic inflammation. PLoS One 8:e81774. doi: 10.1371/journal.pone. 0081774

Veremeyko, T., Yung, A. W. Y., Anthony, D. C., Strekalova, T., and Ponomarev, E. D. (2015). Early growth response gene-2 is essential for M1 and M2 macrophage activation and plasticity by modulation of the transcription factor CEBP $\beta$. Front. Immunol. 9:2515. doi: 10.3389/fimmu.2018.02515

Visvanathan, J., Lee, S., Lee, B., Lee, J. W., and Lee, S.-K. (2007). The microRNA miR-124 antagonizes the anti-neural REST/SCP1 pathway during embryonic CNS development. Genes Dev. 21, 744-749. doi: 10.1101/gad.1519107

Zhang, J., Li, S., Li, L., Li, M., Guo, C., Yao, J., et al. (2015). Exosome and exosomal microRNA: trafficking, sorting, and function. Genom. Proteom. Bioinforma 13, 17-24. doi: 10.1016/j.gpb.2015.02.001

Zhao, X., Wang, H., Sun, G., Zhang, J., Edwards, N. J., and Aronowski, J. (2015). Neuronal interleukin-4 as a modulator of microglial pathways and ischemic brain damage. J. Neurosci. 35, 11281-11291. doi: 10.1523/JNEUROSCI.168515.2015

Conflict of Interest: The authors declare that the research was conducted in the absence of any commercial or financial relationships that could be construed as constituting a potential conflict of interest.

Copyright (c) 2019 Veremeyko, Yung, Dukhinova, Strekalova and Ponomarev. This is an open-access article distributed under the terms of the Creative Commons Attribution License (CC BY). The use, distribution or reproduction in other forums is permitted, provided the original author(s) and the copyright owner(s) are credited and that the original publication in this journal is cited, in accordance with accepted academic practice. No use, distribution or reproduction is permitted which does not comply with these terms. 